\title{
Traveling without dwelling: Extending the timescale accessible to molecular dynamics simulation
}

\author{
Tetsuya Morishita $\odot^{1,2, *}$ and Atsushi M. Ito ${ }^{3}$ \\ ${ }^{1}$ Research Center for Computational Design of Advanced Functional Materials (CD-FMat), \\ National Institute of Advanced Industrial Science and Technology (AIST), 1-1-1 Umezono, Tsukuba 305-8568, Japan \\ ${ }^{2}$ Mathematics for Advanced Materials Open Innovation Laboratory (MathAM-OIL), AIST, c/o AIMR, \\ Tohoku University, 2-1-1 Katahira, Aoba-ku, Sendai 980-8577, Japan \\ ${ }^{3}$ National Institute for Fusion Science, 322-6 Oroshi-cho, Toki 509-5292, Japan
}

(Received 25 June 2019; published 18 October 2019)

\begin{abstract}
Molecular dynamics (MD) simulations have been widely recognized as a promising tool for investigating dynamical processes with an atomic resolution. The time span accessible to MD simulations is, however, extremely limited, which makes it difficult to simulate dynamical events on a timescale of milliseconds or longer while maintaining the microscopic-level resolution. Here, we present an approach that allows us to accelerate the dynamical processes (infrequent events) by introducing an additional bias potential and to describe these events on the correct timescale. To this end, we utilize a reparametrization of time (generalized Poincaré time transformation), which leads to the accelerated dynamics and the biased sampling in phase space. The direct link between the timescale and biased sampling demonstrated in this study makes it possible to extend the timescale in MD simulations without invoking the approximation of the transition state theory. A general form of the bias potential is also introduced, which is easily constructed and straightforwardly applied to various systems. The present approach, Poincaré boost dynamics, is applied to the diffusion process of an impurity atom in an amorphous matrix and to conformational changes of a model protein for demonstrating its validity and versatility.
\end{abstract}

DOI: 10.1103/PhysRevResearch.1.033032

\section{INTRODUCTION}

Molecular dynamics (MD) simulations have been recognized as one of the most powerful tools for investigating dynamical processes at an atomistic level, such as impurity diffusion in a crystal and protein conformational changes. It is, however, well known that MD simulations often suffer from a severe limitation associated with the timescale [1-4]. With current computer resources, MD simulations using a standard protocol for sampling the canonical distribution cannot simulate dynamical processes taking place on a timescale of milliseconds or longer.

Such processes or events often take place only when the system is activated and overcomes energy barriers, which dominantly characterizes the timescale of the events. A variety of biased sampling techniques have thus been introduced in MD simulations, such as multicanonical MD [5-7], accelerated MD [8,9], and Tsallis dynamics [10-12], to increase the rate of overcoming the barriers and to sample such events more frequently. To this end, a bias potential function $\Delta \Phi$ is introduced in addition to the original potential of $\Phi$, leading to the sampling of the distribution of $\exp [-\beta(\Phi+\Delta \Phi)]$, i.e., biased sampling. While most of these techniques

\footnotetext{
*t-morishita@aist.go.jp

Published by the American Physical Society under the terms of the Creative Commons Attribution 4.0 International license. Further distribution of this work must maintain attribution to the author(s) and the published article's title, journal citation, and DOI.
}

succeed in increasing the occurrence rate of such events, their intrinsic timescales, however, cannot be assessed in general, because the dynamics of the system is no longer described by Newtonian-type dynamics on the landscape of $\Phi$.

Voter introduced hyperdynamics $[13,14]$ that allows for accelerating the sampling of the infrequent events and also for estimating the original timescale of these events (such as escape rates for stable states). This is, however, based on transition state theory (TST) [15]; thus the method can only be applied to specific systems where the dynamical processes can be described by TST well. In addition, the way to design $\Delta \Phi$ for hyperdynamics is not straightforward and $\Delta \Phi$ may need to be constructed in an ad hoc manner for each accelerated system [16,17]. More general forms of $\Delta \Phi$ have also been proposed [8]; however, only a few attempts to estimate the dynamical behavior under such a biased potential have been made: these attempts are validated only under specific conditions [18] or extrapolated unbiased conditions [19].

In this paper, we introduce an approach that enables us both to accelerate the dynamical processes and to describe the dynamics on the correct timescale. This approach is not based on TST; thus it is quite general and its applicability is wider than that of TST-based methods. Also, a simple and general form of $\Delta \Phi$ will be introduced, which makes the implementation of our "boost MD" easier. We focus on energy-driven events that can be accelerated by modulating the landscape of $\Phi$; "rare" events that are induced by overcoming "free-energy" or "entropic" barriers are out of the present scope, which would preferably be tackled by a different approach such as $\operatorname{LogMFD}[20,21]$. 
The paper is organized as follows: In Sec. II, a generalized version of the Poincaré time transformation is introduced, based on which our approach is built up without the TST approximation. A general form of the biased potential conforming to the approach is also introduced. In Sec. III, the present approach is validated by applying it to three systems, including amorphous silicon (a-Si) with an impurity atom and coarse-grained models for protein G. Finally, concluding remarks are drawn in Sec. IV.

\section{POINCARÉ BOOST DYNAMICS}

\section{A. Generalized Poincaré time transformation}

Let us consider an $N$ particle system coupled to a heat bath, which is assumed to be described by the Nosé-Poincaré (NP) Hamiltonian [22], $H_{\mathrm{NP}}$,

$$
H_{\mathrm{NP}}=s\left[\sum_{i=1}^{3 N} \frac{\pi_{i}^{2}}{2 m_{i} s^{2}}+\Phi(q)+\frac{p_{s}^{2}}{2 Q}+g k_{B} T \ln s-H_{0}\right] \equiv 0,
$$

where $q_{i}, \pi_{i}$, and $m_{i}$ are the position, "virtual" momentum, and mass of particle $i$, respectively, and $s, p_{s}$, and $Q$ are the thermostat variable, its corresponding momentum, and mass, respectively. $k_{B}$ is Boltzmann's constant, $T$ is the preset temperature, and $g$ is the number of degrees of freedom. $H_{0}$ is a constant to be set to ensure $H_{\mathrm{NP}} \equiv 0$. It is proven that the trajectories from $H_{\mathrm{NP}}$ sample the canonical distribution of $e^{-\beta \Phi}$ in the $q$ space [22], where $\beta=1 / k_{B} T$. The equations of motion (EOMs) from $H_{\mathrm{NP}}$ (for $q_{i}$ and $\pi_{i}$ ) are derived as

$$
\begin{aligned}
\frac{d q_{i}}{d t_{c}} & =\frac{\partial H_{\mathrm{NP}}}{\partial \pi_{i}}, \\
\frac{d \pi_{i}}{d t_{c}} & =-\frac{\partial H_{\mathrm{NP}}}{\partial q_{i}},
\end{aligned}
$$

where $t_{c}$ indicates the timescale on which the original system of $H_{\mathrm{NP}}$ evolves.

Now a Poincare time transformation [23] is applied to the system with the scaling factor $\sigma$,

$$
\sigma(q)=\exp [\beta \Delta \Phi(q)],
$$

where $\Delta \Phi \geqslant 0$, being a function of $q$. We do not specify the explicit form of $\Delta \Phi$ for the moment, which will be discussed later. Poincaré transformation constructs a new Hamiltonian by scaling the original Hamiltonian $H$ by $\sigma$, where $H$ is conserved to zero by adjusting the energy origin. Here, the Hamiltonian of $H_{\mathrm{NP}}$ is transformed to $\sigma H_{\mathrm{NP}}$ by the Poincaré transformation, which results in reparametrization of time as follows [23]:

or

$$
\frac{d t_{c}}{d \tau}=\sigma
$$

$$
d t_{c}=\sigma d \tau
$$

That is, the dynamical variables of $\sigma H_{\mathrm{NP}}$ evolve in the scaled time $\tau$. The EOMs for $q_{i}$ and $\pi_{i}$ from $\sigma H_{\mathrm{NP}}$ are

$$
\begin{aligned}
\frac{d q_{i}}{d \tau} & =\frac{\partial\left(\sigma H_{\mathrm{NP}}\right)}{\partial \pi_{i}}, \\
\frac{d \pi_{i}}{d \tau} & =-\frac{\partial\left(\sigma H_{\mathrm{NP}}\right)}{\partial q_{i}} .
\end{aligned}
$$

In fact, the trajectories from $H_{\mathrm{NP}}$ and $\sigma H_{\mathrm{NP}}$ in phase space (including $s$ and $p_{s}$ ) are identical (at least theoretically), but the Poincaré transformation alters the timescale: the transformed system evolves at a different speed $(\tau)$ along the trajectory due to the time reparametrization [23].

In the meantime, the trajectories from $\sigma H_{\mathrm{NP}}$ sample the canonical distribution of $\exp [-\beta(\Phi+\Delta \Phi)]$ as long as the system is ergodic. This indicates that the Poincarétransformed system enables us to perform the biased sampling in $q$ space,

$$
\begin{aligned}
Z_{\sigma}= & \int d p_{s} d s d \pi d q \delta\left(\sigma H_{\mathrm{NP}}\right) \\
= & \int d p_{s} d s d \pi d q \sigma^{-1} \delta\left(H_{\mathrm{NP}}\right) \\
= & \int d p_{s} d s d p d q \sigma^{-1} s^{3 N-1} \\
& \times \delta\left[\sum_{i=1}^{3 N} \frac{p_{i}^{2}}{2 m_{i}}+\Phi+\frac{p_{s}^{2}}{2 Q}+g k_{B} T \ln s-H_{0}\right] \\
\propto & \int d p d q \exp \left[-\beta\left(\sum_{i=1}^{3 N} \frac{p_{i}^{2}}{2 m_{i}}+\Phi+\Delta \Phi\right)\right]
\end{aligned}
$$

where $Z_{\sigma}$ is the microcanonical partition function for $\sigma H_{\mathrm{NP}}$ with $g=3 N$, and $p_{i}=\pi_{i} / s$ is the "real" momentum of particle $i$ [22] (recall that $\delta[f(s)]=\delta\left(s-s_{0}\right) /\left[d f\left(s_{0}\right) / d s\right]$, where $f(s)$ is a smooth function having a single root at $\left.s=s_{0}\right)$. While most of the biased sampling methods utilize the trajectories on the landscape of $\Phi+\Delta \Phi$ generated by a thermostatted EOM (i.e., $\dot{p}=-\frac{\partial}{\partial q}(\Phi+\Delta \Phi)+$ thermostat), the time reparametrization enables the biased sampling without invoking the dynamics on such a modified landscape (note that we refer to the trajectory that realizes the biased sampling as a "biased trajectory," irrespective of the algorithm to generate it).

What is demonstrated above indicates that the biased sampling [Eq. (9)] results from the reduced dwell time in lowenergy states, which comes from the increased traveling speed along the trajectory ( $\tau$ for the biased trajectory). This, in turn, indicates that, by performing the time reparametrization from $\tau$ to $t_{c}$ for the biased trajectory from $\sigma H_{\mathrm{NP}}$, one can obtain a trajectory that samples the canonical distribution of $e^{-\beta \Phi}$, along which the unbiased system evolves on the "correct" timescale $t_{c}$. This is a key finding that allows the estimate of the correct timescale using the biased trajectories.

This finding actually applies to non-Hamiltonian dynamics with time reparametrization as well (see Appendix A for the case of the Nosé-Hoover (NH) dynamics [24]). While the Poincaré transformation is introduced in the framework of Hamiltonian dynamics [23], the same effect is brought about by the time transformation in non-Hamiltonian dynamics. We therefore refer to the time transformation in either Hamiltonian dynamics or non-Hamiltonian dynamics as "generalized Poincaré transformation (gPT)" [25,26], and the dynamics by the EOM derived from gPT [e.g., Eqs. (7) and (8) and Eqs. (A1)-(A4)] as "gPT dynamics."

We have seen that the gPT dynamics enables us both to perform the biased sampling and to recover the original 
timescale. The point is that, using the gPT dynamics, the relation of Eq. (5) or (6) can be exploited to recover the original (correct) timescale without invoking TST. Hereafter, we call the present approach "Poincaré boost dynamics (PBD)."

A brief remark is in order. The discussion thus far is based on the assumption that the system is ergodic. That is, the trajectory from the biased MD run is assumed to sample the phase-space points that would also be sampled by a sufficiently long unbiased canonical MD trajectory with $\Phi$. It is expected, in general, that a sufficiently long MD trajectory is able to sample rare events without a biased potential. This is the reason why the biased trajectory based on the gPT dynamics samples the rare events more frequently than the unbiased trajectory comprising the same number of MD steps (i.e., the biased trajectory can be seen as a very long unbiased trajectory after the time reparametrization from $\tau$ to $t_{c}$ ). It is, therefore, important in practical biased MD simulations whether the canonical distribution of $e^{-\beta \Phi}$ is recovered by reweighting the phase-space points along the biased trajectory with $\sigma$. We will come back to this point again later. Note that the recovery of the original distribution is also a key to the implementation of the TST-based methods, which is, however, not explicitly pointed out in the literature.

\section{B. Ensemble and time averages for the biased trajectories}

In this subsection, we will discuss in the light of numerical calculations the relation between ensemble averages and the time averages along the biased trajectory.

Consider the canonical ensemble average of $A,\langle A\rangle_{c}$, which is a function of $q$ and is obtained by reweighting the biased MD trajectory as

$$
\begin{aligned}
\langle A(q)\rangle_{c} & =\frac{\int d q A(q) e^{\beta \Delta \Phi} e^{-\beta \Phi} e^{-\beta \Delta \Phi}}{\int d q e^{-\beta \Phi} e^{-\beta \Delta \Phi}} / \frac{\int d q e^{\beta \Delta \Phi} e^{-\beta \Phi} e^{-\beta \Delta \Phi}}{\int d q e^{-\beta \Phi} e^{-\beta \Delta \Phi}} \\
& =\frac{\left\langle A e^{\beta \Delta \Phi}\right\rangle_{\Delta \Phi}}{\left\langle e^{\beta \Delta \Phi}\right\rangle_{\Delta \Phi}},
\end{aligned}
$$

where \langle\rangle$_{\Delta \Phi}$ indicates a canonical ensemble average using the biased trajectory. If the system is ergodic, we can invoke the equivalence between an ensemble average $\langle A\rangle_{\Delta \Phi}$ and a time average $\bar{A}$ on the timescale $(\tau)$ along the biased trajectory. The canonical average of Eq. (10) can thus be numerically computed as

$$
\begin{aligned}
\frac{\left\langle A e^{\beta \Delta \Phi}\right\rangle_{\Delta \Phi}}{\left\langle e^{\beta \Delta \Phi}\right\rangle_{\Delta \Phi}} & =\frac{\overline{A e^{\beta \Delta \Phi}}}{\overline{e^{\beta \Delta \Phi}}}=\frac{\lim _{t \rightarrow \infty} \frac{1}{t} \int^{t} A(\tau) e^{\beta \Delta \Phi(\tau)} d \tau}{\lim _{t \rightarrow \infty} \frac{1}{t} \int^{t} e^{\beta \Delta \Phi(\tau)} d \tau} \\
& \simeq \frac{\sum_{i}^{N_{s}} A_{i} e^{\beta \Delta \Phi_{i}} \Delta \tau}{N_{s} \Delta \tau} / \frac{\sum_{i}^{N_{s}} e^{\beta \Delta \Phi_{i}} \Delta \tau}{N_{s} \Delta \tau} \\
& =\sum_{i} A_{i} \Delta t_{c}^{i} / \sum_{i} \Delta t_{c}^{i},
\end{aligned}
$$

where $N_{s}$ is the total number of time steps, $A_{i}=A\left[q\left(n_{i} \Delta \tau\right)\right]$, $\Delta \Phi_{i}=\Delta \Phi\left[q\left(n_{i} \Delta \tau\right)\right]$, and

$$
\Delta t_{c}^{i}=e^{\beta \Delta \Phi_{i}} \Delta \tau \text {. }
$$

$\Delta \tau$ and $\Delta t_{c}$ are the time step for the biased and unbiased MD trajectories, respectively, and the relation of Eq. (12) is obtained by invoking $d t_{c}=e^{\beta \Delta \Phi} d \tau$ (one can only argue that $\Delta t_{c}$ is proportional to $e^{\beta \Delta \Phi} \Delta \tau$ without $d t_{c}=e^{\beta \Delta \Phi} d \tau$. See also Sec. II C for a relevant discussion).

Equation (11) demonstrates that the canonical average of $A$ can be evaluated as the time average on the timescale $t_{c}$. In other words, the time averages along the biased MD trajectory using Eq. (12) are equivalent to the unbiased canonical ensemble averages. The total time the system of $\Phi$ evolved in the corresponding unbiased MD run is estimated as

$$
T_{c}=\sum_{i}^{N_{s}} \Delta t_{c}^{i}=\sum_{i}^{N_{s}} e^{\beta \Delta \Phi_{i}} \Delta \tau .
$$

Using $T_{c}$, for example, average escape time (average time for escape from a certain state) in the original unbiased system can be evaluated as $T_{c} / N_{\text {esc }}$, where $N_{e s c}$ is the effective number of attempted escapes [see also Eq. (21)].

The relation of Eq. (12) was previously introduced in hyperdynamics $[13,14]$, but we have shown that this relation can be employed without the assumption of TST. The discussion here is quite general and is actually valid not only for the gPTbased MD calculations but also for standard thermostatted MD calculations wherein the biased trajectories are generated. This will be discussed in more detail in Sec. II D.

\section{Remarks on the practical implementation}

There are two remarks on running the biased MD calculations. First, a relatively small $\Delta \tau$ may be required in biased MD runs using the gPT dynamics. This is because the atomic dynamics is often accelerated by the modified force with $\sigma$, which could become large due to the exponential factor. The numerical stability in integrating the gPT-based EOM should thus be carefully examined.

The secondary (and more important) issue is related with the arbitrariness of the scaling factor $\sigma$. Consider $g \sigma(g>0)$ as the scaling factor of the Poincaré time transformation. We have in this case $d t_{c}=g \sigma d \tau$, but this relation still allows us to estimate the canonical ensemble average of $A$ correctly [Eq. (10)]. One thus might consider employing $g \sigma$ instead of $\sigma$ for the timescaling factor in estimating the dynamical properties on $\Phi$. This indicates that the unit of time cannot be uniquely determined from the biased trajectory only.

To remove this arbitrariness, we require $\sigma=1$ (i.e., $\Delta \Phi=$ $0)$ when $\Phi>\varepsilon_{0}$, where $\varepsilon_{0}$ is high enough to boost the dynamics we are interested in, but is lower than the highest energy sampled in the biased MD run. As long as $\Delta \Phi$ (and the force from it) is smoothly connected to 0 as $\Phi$ approaches $\varepsilon_{0}$, the dynamics along the resultant biased trajectory shows no discontinuous behavior, and we can correctly estimate the dynamical behavior on $\Phi$ using PBD with the uniquely determined $\sigma$ (this condition also obviates a sudden change of $\Delta t_{c} / \Delta \tau$ at $\left.\Phi=\varepsilon_{0}\right)$ [27].

\section{Standard thermostatted dynamics for the biased MD runs}

In the PBD approach, the gPT dynamics is utilized for generating the biased trajectories. It should be, however, pointed out that the gPT dynamics has some shortcomings in its practical implementation. While the accelerated sampling by the gPT dynamics is highly effective when the time step and the 
number of MD steps are the same as used in the unbiased MD runs, the time step $\Delta \tau$ may need to be relatively small as mentioned before, which could reduce the advantage of the gPT dynamics. In addition, numerical integrations of the specific EOM derived from gPT may not be straightforward, which would require a complicated integration algorithm [22,23,28]. One may thus wonder if the thermostatted dynamics other than the gPT dynamics can also be utilized for generating the biased trajectories in PBD. In other words, the question is whether the trajectory by other thermostatted dynamics can be a substitute for the gPT-based trajectory. Although the former is no longer identical to the original unbiased trajectory in phase space, it can still serve as the biased trajectory in PBD as discussed below.

The second remark in the previous subsection suggests that, as long as the energy-driven dynamics is the focus of interest (e.g., average escape time), the standard thermostatted dynamics such as the NH dynamics on the landscape of $\Phi+\Delta \Phi$

$$
\begin{gathered}
\frac{d q_{i}}{d \tau}=\frac{p_{i}}{m_{i}}, \\
\frac{d p_{i}}{d \tau}=-\frac{\partial}{\partial q_{i}}(\Phi+\Delta \Phi)-p_{i} \frac{p_{\eta}}{Q}, \\
\frac{d \eta}{d \tau}=\frac{p_{\eta}}{Q}, \\
\frac{d p_{\eta}}{d \tau}=\left[\sum_{i=1} \frac{p_{i}^{2}}{m_{i}}-g k_{B} T\right],
\end{gathered}
$$

can also be employed to generate the biased trajectories ( $\eta$ and $p_{\eta}$ are the thermostat variable and its momentum, respectively). The key to the use of the standard dynamics is the reproducibility of the correct dynamics and the canonical distribution in the unbiased simulations. In fact, the dynamical properties obtained using the NH dynamics and the Gaussian isokinetic (GIK) dynamics [29], as well as the NP dynamics, are coincident with those using Newton's dynamics in the limit $N \rightarrow \infty[30,31]$. These dynamics therefore correctly reproduce the timescale when $\Delta \Phi=0$, being the standard timescale [32]. As the correction term for the timescale, $\sigma$, is uniquely determined by referring to the standard timescale, the total dwell time in each energy state can be correctly estimated using $\Delta t_{c}=\sigma \Delta \tau$ even when $\Delta \Phi>0$, provided that the canonical distribution of $e^{-\beta \Phi}$ is recovered with $\sigma$. Once the dwell time in each state is obtained, the total time the system evolved can also be estimated, which is a prerequisite for estimating, e.g., the average escape time. The standard dynamics such as the NH dynamics [Eqs. (14)-(17)], therefore, allow us to describe the energy-driven dynamics on the correct timescale.

In this line, the Langevin dynamics [33] and the Berendsen's weak coupling dynamics [34] are not recommended for generating the biased trajectories. The former may not give the correct dynamics even without a bias potential [35], while the latter does not reproduce the canonical distribution [36].

It should be remarked here that, using the standard dynamics with $\Phi+\Delta \Phi$, short-time dynamics of an individual atom is unlikely to be recovered "correctly." This is because the time step estimated by $\Delta t_{c}=e^{\beta \Delta \Phi_{i}} \Delta \tau$ may become quite large, which is not suitable for describing the individual shorttime dynamics. Rather, we should see that we have a (partly) coarse-grained trajectory in time, which still samples the canonical distribution of $e^{-\beta \Phi}$. Therefore, only the dynamical properties averaged over the trajectories (or segments of the trajectory) are considered to be correctly estimated.

This remark is related to the fact that the biased trajectory based on the standard dynamics is no longer identical to the unbiased trajectory in contrast to the gPT-based trajectory. Even sequences of some dynamical events could differ from those in the unbiased trajectory. Once the original canonical distribution is recovered using $\sigma$, however, the dwell time in each energy state can be correctly estimated (see also Sec. II B). In Sec. III, we will demonstrate that the standard $\mathrm{NH}$-type dynamics indeed works in the framework of PBD. Note, in passing, that, although the trajectories by the standard dynamics are irrelevant to the Poincaré transformation, we also refer to the approach using the standard dynamics as PBD, since it shares the basic framework with the gPT-based approach in Sec. II A (except for the procedure to reproduce the biased trajectories).

\section{E. Bias potential}

We now discuss the explicit form of $\Delta \Phi(q)$. In order to boost the dynamical events, $\Delta \Phi$ should be introduced so as to increase the occurrence frequency of the events, but not to impede the relevant dynamics in the unbiased MD trajectory nor to alter the underlying mechanism $[13,14]$. These are general requirements for $\Delta \Phi$, particularly in non-gPT standard dynamics, irrespective of its explicit form. In addition, we assume that the events we are interested in are well described by the degrees of freedom of $\boldsymbol{x}$ and that these are accelerated by $\boldsymbol{x}$ 's frequent barrier crossing of the energy barrier on $\Phi$. We do not explicitly specify $\boldsymbol{x}$ here, but it may be related with, for example, the torsional degrees of freedom in the case of biomolecule systems $[8,37]$. We thus require that $\Delta \Phi$ greatly depends on $\boldsymbol{x}$, i.e., $\Delta \Phi(\boldsymbol{x} ; q)$. Otherwise, the dynamics of $\boldsymbol{x}(\boldsymbol{x}$ dynamics) would be unaffected and could not be sufficiently accelerated in the case of non-gPT dynamics. (Rather, the dynamics of other degrees of freedom, that are not relevant to the events, could be accidentally accelerated.)

We introduce the following bias potential $\Delta \Phi_{\text {log }}$ :

$$
\Delta \Phi_{\log }=\mu(q)-\frac{1}{\alpha} \log [\alpha \mu(q)+1],
$$

where

$$
\mu(q)=\varepsilon_{0}-\Phi(\boldsymbol{x} ; q),
$$

and $\varepsilon_{0}$ and $\alpha$ are the parameters (in units of energy and its inverse, respectively) that are manually adjusted to efficiently boost the " $\boldsymbol{x}$ dynamics." $\Delta \Phi_{\log }$ depends only on $\Phi(\boldsymbol{x} ; q)$ (part of the $\Phi$, which largely depends on $\boldsymbol{x}$ ) and requires no information on the details of the landscape of $\Phi(x ; q)$ such as the gradient and Hessian with respect to $\boldsymbol{x}$, in contrast to the previously proposed $\Delta \Phi$. The construction of $\Delta \Phi_{\log }$ is thus quite easy. In the case that $\Phi(\boldsymbol{x} ; q)$ is coincident with the whole $\Phi$, the total potential is given as

$$
\Phi+\Delta \Phi_{\log }=\varepsilon_{0}-\frac{1}{\alpha} \log \left[\alpha\left(\varepsilon_{0}-\Phi\right)+1\right] .
$$




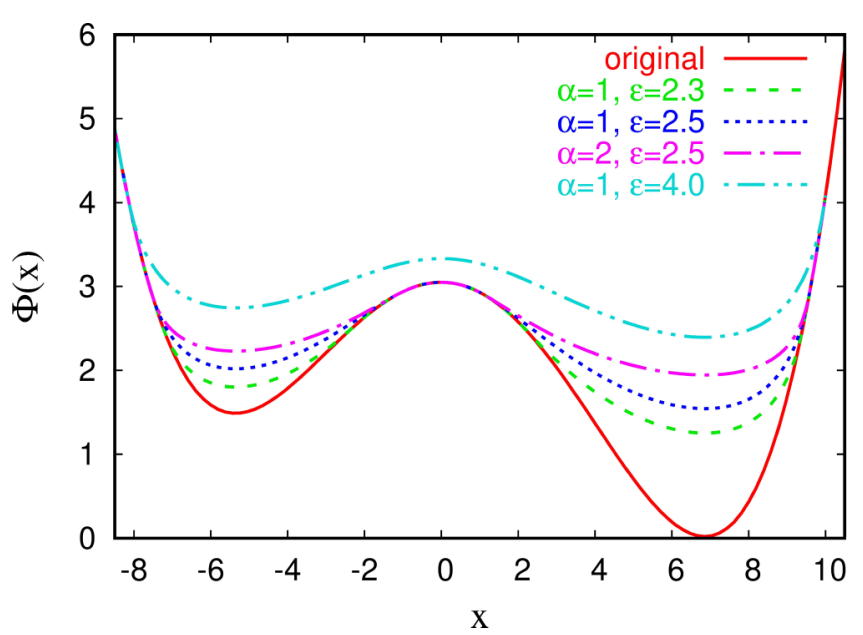

FIG. 1. A schematic one-dimensional potential $\Phi$ and its modified potentials $\left(\Phi+\Delta \Phi_{\log }\right)$ with the parameter sets given in the figure $\left[\Phi(x)=0.0001(2 x-1)^{4}-0.12 x^{2}+3.05\right]$. It is seen that the modification is more aggressive with larger $\alpha$ (compare blue and pink lines) and/or larger $\varepsilon_{0}$ (compare green, blue, and light blue lines).

We easily see that $\Delta \Phi_{\log } \rightarrow 0$ as $\Phi \rightarrow \varepsilon_{0}$ (and its derivative also approaches 0 ). Furthermore, preliminary knowledge of the location of the TST dividing surface and the exact height of the barriers on $\Phi$ is not necessary. Figure 1 shows how the original potential function is modified by adding the bias potential $\Delta \Phi_{\log }$. It is clearly seen that the energy barriers are substantially reduced, which enhances the barrier crossing, and that the modification is more aggressive with larger $\alpha$ and/or $\varepsilon_{0}$ (in Appendix B, we briefly discuss how to set up PBD calculations, including the parameter setting). Note that an alternate form of $\Delta \Phi$ has been proposed by Hamelberg et al. [8], which also conforms to the PBD approach (see also Appendix B for more details).

As discussed earlier, $\varepsilon_{0}$ is set to have $\Delta \Phi_{\log }=0$ when $\Phi>$ $\varepsilon_{0}$, and $\Phi+\Delta \Phi_{\log }$ is smoothly connected to $\Phi$ at $\Phi=\varepsilon_{0}$. It is convenient, in general, to set $\varepsilon_{0}$ below $\varepsilon_{x}$, above which the $\boldsymbol{x}$ 's barrier crossing is triggered (e.g., a diffusion process induced by overcoming energy barriers of $\varepsilon_{x}$ or higher). This requires no correction for the timescale of the focused dynamical processes, since the relevant $\boldsymbol{x}$ dynamics itself (e.g., overcoming the barriers) is described by the standard EOM without the bias potential $\left(\Delta \Phi_{\log }=0\right)$. Although some preliminary knowledge may be necessary to find a reasonable value of $\varepsilon_{x}$, the condition $\varepsilon_{0} \leqslant \varepsilon_{x}$ makes it easy to analyze the biased MD trajectory (we will show in Sec. III B that this approach can be exploited even when the recovery of the distribution $e^{-\beta \Phi}$ is imperfect).

In fact, however, the condition $\varepsilon_{0} \leqslant \varepsilon_{x}$ is not always mandatory in contrast to the TST-based approach [38]. The escape rate, for example, is estimated as follows. The number of escape attempts $N_{e s c}$ can be computed as

$$
N_{e s c}=N_{c}\left\langle\sum_{i} \delta\left(x-x_{i}\right)\right\rangle_{c},
$$

where $x_{i}$ is one of the crossing points that are used to judge if the $\boldsymbol{x}$ trajectory escapes from a basin. $N_{c}$ is the effective total number of the phase-space points available in the unbiased trajectory obtained by reweighting the biased trajectory;

$$
N_{c}=T_{c} / \Delta \tau=\sum_{i}^{N} e^{\beta \Delta \Phi_{i}}
$$

The average escape rate, $k=N_{e s c} / T_{c}$, is thus calculated as

$$
k=N_{c}\left\langle\sum_{i} \delta\left(x-x_{i}\right)\right\rangle_{c} / T_{c},
$$

and the average escape time is

$$
1 / k=T_{c} / N_{e s c}=T_{c} / N_{c}\left\langle\sum_{i} \delta\left(x-x_{i}\right)\right\rangle_{c} .
$$

Equation (21) is valid under the condition of either $\varepsilon_{0} \leqslant \varepsilon_{x}$ or $\varepsilon_{0} \geqslant \varepsilon_{x}$; thus these properties are appropriately estimated in either case (we have confirmed this point in our test calculations for a double-well potential model, wherein $\varepsilon_{0}$ is set above or below the barrier height). If all escape events take place at $\Phi>\varepsilon_{0}, k$ is reduced to $k=N_{e s c}^{0} / T_{c}$, where $N_{e s c}^{0}$ is the actual number of the escape attempts counted in the biased MD run.

\section{APPLICATION}

We will show three illustrative applications of PBD. The following dynamical properties are considered; diffusion coefficients of an impurity atom in a-Si and escape rates associated with structural transformations of a coarse-grained protein model. For the latter, two types of folding-unfolding transitions are considered; one is characterized by the endto-end distance and another is by the radius of gyration. In the biased runs for all these systems, NH-type standard thermostatted dynamics was employed.

\section{A. Impurity migration in a-Si}

We introduce a model system of a-Si containing an impurity atom, wherein the diffusion of the impurity atom is an energy-driven process. The biased and unbiased MD simulations were performed for the system consisting of $511 \mathrm{Si}$ atoms and a single impurity atom in a cubic supercell of $21.758 \AA$. All the interatomic interactions are described by the Stillinger-Weber (SW) potential [39]; the interaction between the Si and impurity atom is also modeled by the SW potential, but only its two-body term scaled by 0.05 is used. The mass of the impurity atom is set to be equal to the mass of a boron (B) atom, although the interaction is not designed to describe the interaction between $\mathrm{Si}$ and "real" B atoms. The impurity, the "B-like" atom, is thus simply a model particle that can diffuse by a jumping process in a-Si. In order to perform long $(\sim 1 \mu \mathrm{s})$ unbiased MD runs at relatively low temperatures for comparison, all the $\mathrm{Si}$ atoms were fixed at their equilibrium positions, while the B-like atom was allowed to diffuse in the a-Si matrix, whose dynamics was described by the standard NH dynamics [Eqs. (14)-(17)]. $\Delta \Phi_{\log }$ thus contains only the potential energy function for the B-like atom, $\Phi_{\mathrm{B}}$. The EOM for the impurity atom was numerically integrated with a time 
step of $1 \mathrm{fs}$, using the algorithm in Refs. [40,41], in both of the biased and unbiased MD runs.

The diffusion coefficient $(D)$ of the B-like atom was evaluated in the biased and unbiased runs, respectively, and is plotted in Fig. 2(a) as a function of inverse temperature. We were able to estimate $D$ at $450-700 \mathrm{~K}$ in the unbiased MD runs; however, no diffusion process was observed below $450 \mathrm{~K}$ even for $1 \mu \mathrm{s}$. In contrast, we were able to evaluate $D$ in the biased MD runs at temperatures down to (at least) $300 \mathrm{~K}$.

Although the jump motion of the B-like atom becomes less frequent and thus the uncertainty of $D$ becomes large as the temperature decreases in the unbiased MD runs, the agreement of $D$ in the biased and unbiased runs is satisfactory. $D$ below $450 \mathrm{~K}$ was evaluated only in the biased runs, but the temperature dependence of the resultant $D$ is reasonable, indicating that PBD correctly predicts the timescale of the diffusion process at low temperatures (note that a similar temperature dependence of impurity diffusion in a-Si is reported in Ref. [42]).

We tried three sets of the parameters for the biased runs, $\left(\alpha, \varepsilon_{0}\right)=(10,-0.25),(20,-0.30)$, and $(30,-0.30)$, and confirmed that the results were not affected by the choice of the parameters. $D$ obtained by different parameter sets is in excellent agreement with each other at all the temperatures. Among these parameter sets, the highest boost factor of 53 is obtained with the set of $\alpha=10$ and $\varepsilon_{0}=-0.25$ at $300 \mathrm{~K}$ (note that energies in the SW model are measured in units of $50 \mathrm{kcal} / \mathrm{mol})$.

As mentioned before, the canonical distribution with $\Phi$ should well be recovered to reproduce the correct timescale. This is indeed confirmed in Fig. 2(b). The distributions of the potential energy of the B-like atom, $P\left(\Phi_{\mathrm{B}}\right)$, at 700 and $500 \mathrm{~K}$ obtained from the unbiased MD trajectories are compared with that from the biased MD trajectories with $\alpha=10$ and $\varepsilon_{0}=-0.25$. It is clearly seen that the $P\left(\Phi_{\mathrm{B}}\right)$ recovered by reweighting the distributions from the biased MD trajectories (triangles) agree well with $P\left(\Phi_{\mathrm{B}}\right)$ from the unbiased trajectories (lines). It is also worth noting that the original $P\left(\Phi_{\mathrm{B}}>\varepsilon_{0}\right)$ in the biased runs (open circles) is highly enhanced by the bias potential of $\Delta \Phi_{\mathrm{log}}$, indicating that the jump motion of the B-like atom was much more frequently induced in the biased MD runs.

The highly diffusive motion of the impurity atom in the biased runs can be visually confirmed in Fig. 2(c), where the position of the B-like atom at 100-fs intervals is shown (during a $0.1-n s$ period at $500 \mathrm{~K}$ ). It is clearly seen that the biased trajectory is highly extended indicating frequent jump motions, while the unbiased trajectory is compacted at a single site showing no diffusion during the time period in Fig. 2(c).

\section{B. Conformational changes of a coarse-grained protein-G model (model 1)}

The second illustration is the transition rate for the foldingunfolding transition of a coarse-grained protein model. The model employed here was originally introduced to describe the transformation mechanism of protein G (PDB id: 2gb1) using a simple bead-spring chain [43]. The model consists of 56 amino acids, each being treated as a united particle. The nonbonded interaction between the united particles can

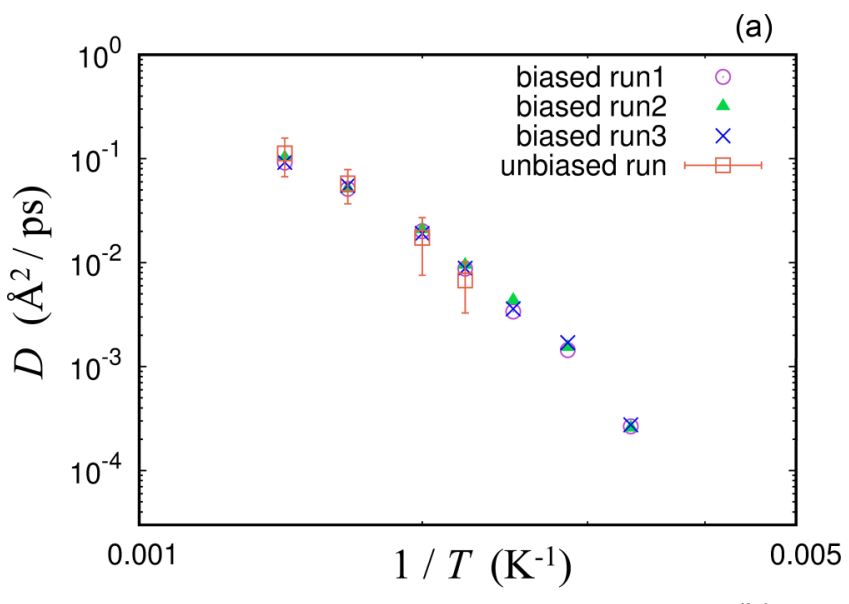

(b)

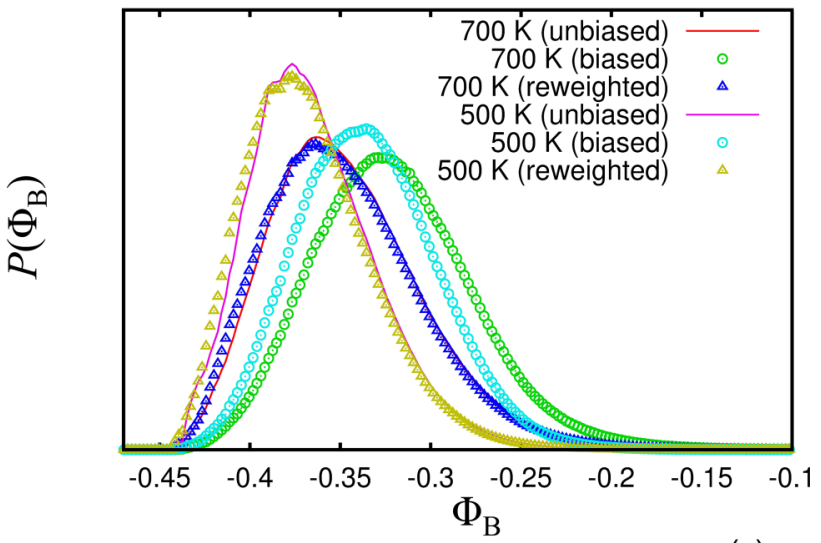

(c)

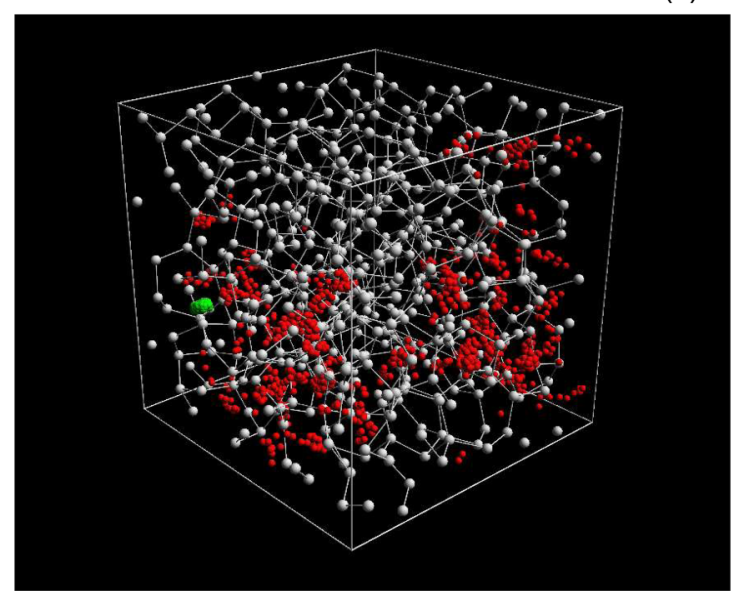

FIG. 2. (a) Diffusion coefficient $D$ of the B-like atom as a function of inverse temperature. $D$ is evaluated in the three biased trajectories, each employing a different parameter set, and one unbiased trajectory, all being in agreement with each other. (b) Distribution of $\Phi_{\mathrm{B}}$ for the 700 and $500 \mathrm{~K}$ runs. (c) Diffusion processes of the B-like atom in amorphous $\mathrm{Si}$. Trajectory of the B-like atom is denoted by a series of 1000 red (green) particles for the biased (unbiased) run, each indicating its position at 100 -fs intervals during a 0.1 -ns period at $500 \mathrm{~K}$. The Si atoms are represented by the white particles, and thin white lines indicate the supercell.

be tuned to exert various solvation effects by employing the Lennard-Jones (LJ) interaction and Coulomb interaction. Several parameter sets for these interactions were tested in 
Ref. [43]. Here we employ two parameter sets; one is for the transition described by the end-to-end distance $l_{d}$ (first model) and another is for the transition described by the radius of gyration $R_{g}$ (second model) [44]. In the first model, the interaction between the terminal united-residue groups is further modified so that the unfolded state $\left(l_{d}>3 \sigma\right)$ is rarely induced in unbiased MD simulations. Lengths and energies of the protein model are measured in units of $\sigma=$ $3.8 \AA$ and $\varepsilon=3 \mathrm{kcal} / \mathrm{mol}$, respectively. In both of the biased and unbiased MD runs, two Nosé-Hoover heat baths are recursively attached to each of the united particles to sample the canonical distribution (massive recursive Nosé-Hoover approach [45-47]). The EOM for the recursive NH dynamics was numerically integrated with a time step of $5 \mathrm{fs}$, using the algorithm in Ref. [46].

Figure 3(a) shows the profile of the interaction energy (potential energy $\Phi_{l d}$ ) between the terminal groups in the first model. The interaction is modified to be stronger than the original one to keep $l_{d}$ short, making the model prefer a compact folded state. Also shown is the profile with the biased potential, $\Phi_{l d}+\Delta \Phi_{\log }^{l d}$, with which we ran biased MD simulations to enhance the occurrence of the transition from the folded to unfolded (elongated) states having $l_{d}>3 \sigma$ (i.e., only the $\Phi_{l d}$ part of the whole $\Phi$ is modified with $\Delta \Phi_{\log }^{l d}$ ). The parameters for $\Delta \Phi_{\log }^{l d}$ were set as $\alpha=1$ and $\varepsilon_{0}=-3$.

The time evolution of $l_{d}$ in the biased and unbiased MD runs for the first model is shown in Fig. 3(b). We clearly see that, while $l_{d}$ in the unbiased run rarely exceeds $3 \sigma$, it frequently exceeds $3 \sigma$ in the biased run, indicating that the transition is frequently induced by PBD. The transition (escape) rate from the folded state is estimated as a function of inverse temperature (220-300 K) in Fig. 3(c). The agreement between the biased and unbiased results is excellent at 260$300 \mathrm{~K}$. While no unfolding transition was induced in the unbiased run below $260 \mathrm{~K}$ (even for the $3 \times 10^{9}$ steps run), it was successfully reproduced in the biased MD run down to $220 \mathrm{~K}$. We found that, in this application, the boost factor ranges from 35 to 164 (depending on the temperature). Also, we confirmed that the $l_{d}$ distribution in the (unbiased) original system was well recovered by reweighting the biased MD trajectories using $\Delta \Phi_{\log }^{l d}$ (not shown), resulting in the reliable estimation of the transition rate at various temperatures.

\section{Conformational changes of a coarse-grained protein-G model (model 2)}

Another type of folding-unfolding transition demonstrated in this section is characterized by $R_{g}$. In fact, $R_{g}$ depends on all degrees of freedom in the protein model, which often raises a problem associated with the reweighting. While we have addressed the importance of the recovery of the canonical distribution by reweighting, it is sometimes difficult to reproduce the canonical distribution in the whole energy range relevant to the system [48]. This is often encountered, especially when the boost factor is too aggressive or the boosted part of the system consists of a large number of degrees of freedom $(\boldsymbol{x})$. The correct timescale $t_{c}$ is, in principle, no longer estimated in this case; however, the total time the unbiased system evolved, $T_{c}$, can still be evaluated when certain conditions are fulfilled. We will show, using the second protein model, how to tackle this problem.

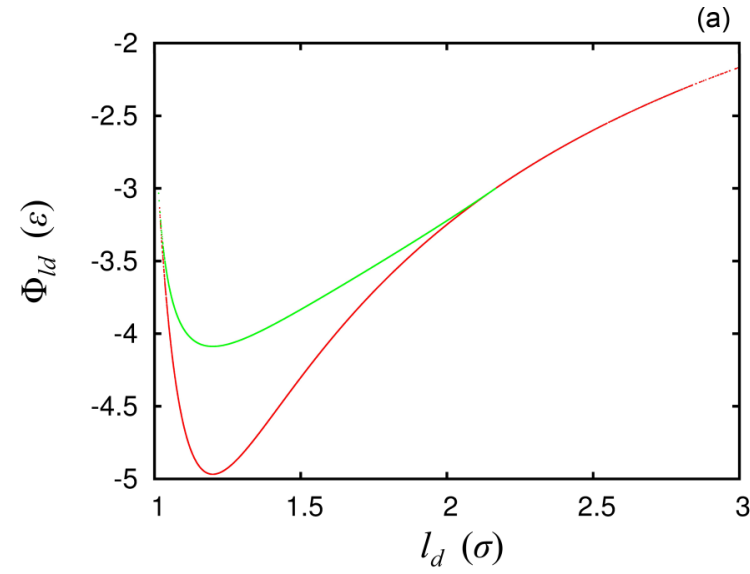

(a)
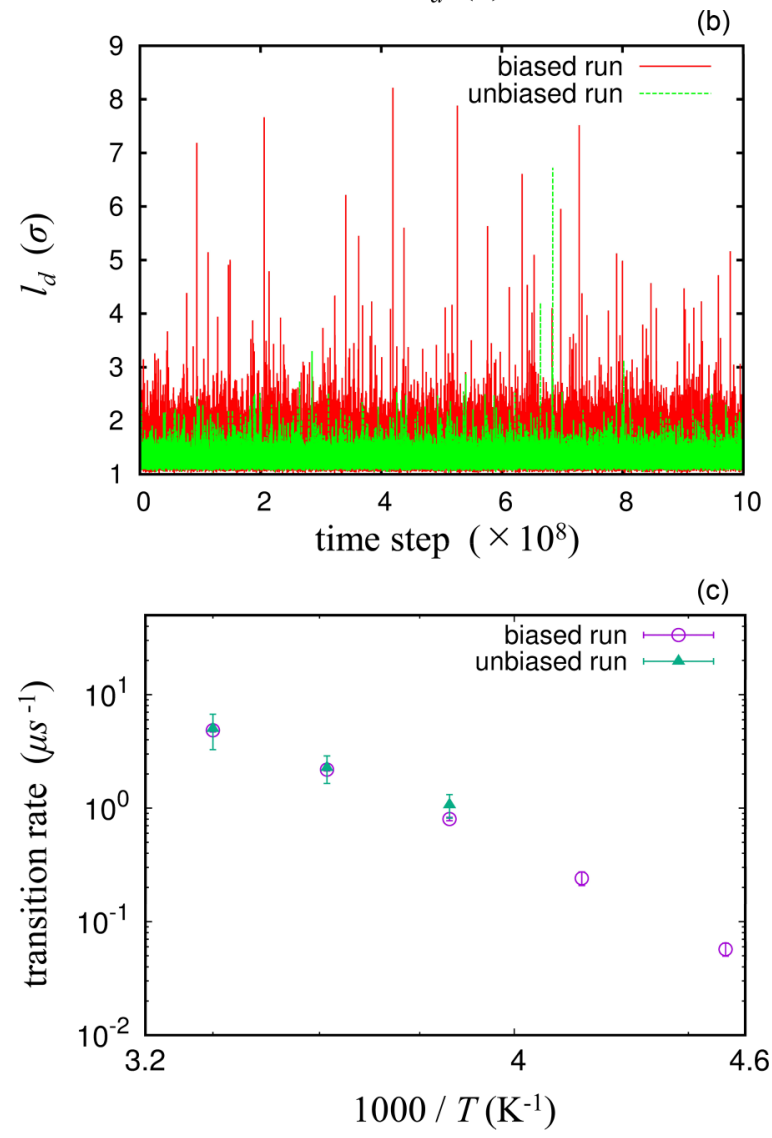

FIG. 3. (a) Potential profile for the interaction between the terminal groups in the protein-G model (model 1). The original potential $\left(\Phi_{l d}\right)$ is shown by the red curve, while the modified potential $\left(\Phi_{l d}+\right.$ $\Delta \Phi_{\log }^{l d}$ ) is shown by the green curve. (b) Time evolution of end-to-end distance $\left(l_{d}\right)$ in the biased and unbiased runs. (c) Transition (escape) rate from the folded state as a function of inverse temperature.

We assume that the event we want to frequently sample is triggered only when $\Phi>\varepsilon_{0}$ (where $\Delta \Phi=0$ ) and that the range $\Phi>\varepsilon_{0}$ is well sampled by the biased trajectory. This indicates that the biased trajectory samples the canonical distribution of $\exp [-\beta \Phi]$ in the energy range above $\varepsilon_{0}$. Furthermore, the phase-space points having $\Phi \leqslant \varepsilon_{0}$ are assumed to be well sampled using an unbiased MD trajectory. The canonical distribution in the whole energy range can therefore be constructed by combining the sampled data in the biased 


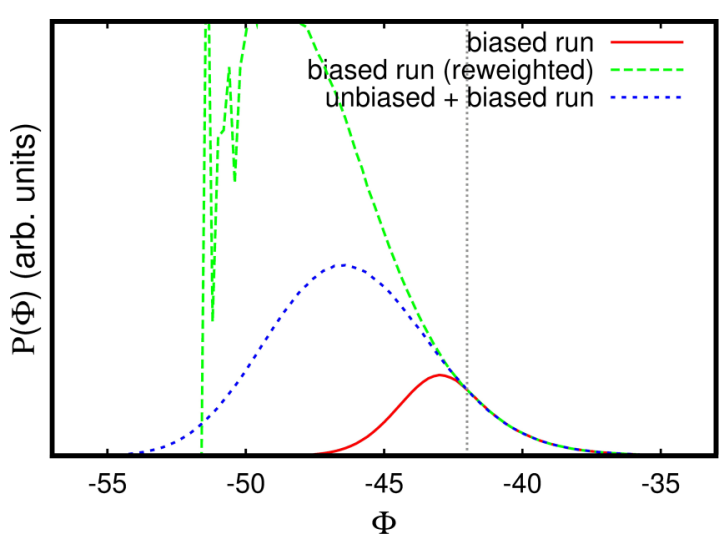

FIG. 4. Distribution of the whole potential $P(\Phi)$ from the biased and unbiased runs. $P(\Phi)$ obtained by reweighting the $P(\Phi)$ from the biased run is shown by the green dashed lines. The blue dotted line represents $P(\Phi)$ which is obtained by combining the $P\left(\Phi \leqslant \varepsilon_{0}\right)$ from the unbiased run and the $P\left(\Phi>\varepsilon_{0}\right)$ from the biased run, where $\varepsilon_{0}$ is -42 (indicated by the vertical dotted line). Note that each $P(\Phi)$ is scaled to have the same intensity at $\varepsilon_{0}(=-42)$, so that the distributions are not normalized to have the same area.

and unbiased MD runs. From the discussion in Appendix C, $T_{c}$ can be approximately evaluated as $T_{c}=C_{\text {boost }} T_{c}^{\prime}$, where $C_{\text {boost }}$ is defined in Eq. (C2) (see Appendix C) and $T_{c}^{\prime}$ is the "actual" total time the system evolved in the unbiased MD run (the exact expression for $T_{c}$ is given in Appendix C).

Using $T_{c}$ evaluated in this way, we demonstrate that dynamical properties of the second protein model in the unfolded state $\left(R_{g} \geqslant 4\right)$ are correctly estimated. The biased potential $\Delta \Phi_{\log }$ in this system is designed to affect the whole protein chain, so that $R_{g}$ frequently takes a large value $\left(R_{g} \geqslant 4\right)$. Hence, $\mu$ in Eq. (19) contains the whole $\Phi$. Figure 4 shows the distribution of the potential energy of the whole protein. The distribution, $P(\Phi)$, from the unbiased run is uniformly scaled by $C_{\text {boost }}$ to have the same intensity as that from the biased run at $\Phi=\varepsilon_{0}(=-42)[P(\Phi)$ are thus not normalized identically].

It is clearly seen that the combined distribution (blue lines) reproduces the canonical distribution in the whole energy range, which is exploited for evaluating $T_{c}$. Also shown is that the canonical distribution is not recovered in $\Phi \leqslant \varepsilon_{0}$ (green lines) by reweighting the $P\left(\Phi \leqslant \varepsilon_{0}\right)$ from the biased MD run, which is often the case when many degrees of freedom are included in $\Delta \Phi_{\log }$. The unfolded state $\left(R_{g} \geqslant 4\right)$, however, was found to be induced only when the potential energy exceeded $\sim-40$. As long as $\varepsilon_{0}$ is below -40 , therefore, the dwell time in the unfolded state and the transition rate, for example, can be correctly predicted: The former is evaluated using the data only when $\Delta \Phi_{\log }=0$, and the latter is computed as $k=N_{e s c}^{0} / T_{c}$.

Table I shows these dynamical properties obtained from two biased MD runs using different parameter sets and one unbiased long MD run. We see that the dwell time and the transition rate, respectively, from the three MD runs are in excellent agreement with each other. In particular, the uncertainty of each measurement is substantially reduced in the biased runs, indicating a much more frequent occurrence of the transition. Also, the total time the system evolved $\left(T_{c}\right)$,
TABLE I. Dwell time in the unfolded state $\left(R_{g} \geqslant 4\right)$ and the transition (escape) rate from the folded state for the protein- $\mathrm{G}$ model (model 2).

\begin{tabular}{lcc}
\hline \hline & Dwell time $(\mathrm{ps})$ & Transition rate $\left(\mu \mathrm{s}^{-1}\right)$ \\
\hline $\begin{array}{l}\text { Biased run 1 } \\
\left(\alpha=0.1, \varepsilon_{0}=-42\right)\end{array}$ & $5.89 \pm 0.26$ & $9.55 \pm 1.22$ \\
$\begin{array}{l}\text { Biased run 2 } \\
\left(\alpha=0.3, \varepsilon_{0}=-44\right)\end{array}$ & $5.57 \pm 0.23$ & $9.50 \pm 2.12$ \\
Unbiased run & $5.59 \pm 0.46$ & $10.51 \pm 4.01$ \\
\hline \hline
\end{tabular}

which was used to compute the transition rate, is correctly estimated following the discussion in Appendix C. It is thus concluded that the reweighting problem can be obviated by combining the biased and unbiased MD data in the framework of the PBD approach, when the focused events take place in the range $\Phi>\varepsilon_{0}$.

\section{CONCLUSIONS}

We have introduced an alternative approach, PBD, to tackle the timescale problem in molecular dynamics simulations. In order to estimate the correct timescale of the dynamical events induced in biased MD runs, we utilized a generalized version of the Poincaré time transformation $(\mathrm{gPT})$. Since the trajectories in phase space are not altered but the traveling speed only is altered by $\mathrm{gPT}$, correct dynamical behavior is recovered without invoking TST. While the gPT dynamics is rather theory driven and may not always be suitable for practical calculations, standard thermostatted dynamics such as the NH dynamics can also be employed to produce the biased trajectories, as long as the energydriven dynamics is the focus of interest. The keys to the estimate of the correct timescale, which are crucial ingredients of PBD, are as follows: (1) $\sigma=1$ is ensured in the range $\Phi>\varepsilon_{0}$, and (2) the canonical distribution for the original potential is recovered with $\sigma$. These features validate the time reparametrization, $\Delta t_{c}=e^{\beta \Delta \Phi_{i}} \Delta \tau$, for the biased trajectories from various thermostatted dynamics. Even in the case that the recovery of the original canonical distribution fails, the present approach still allows us to evaluate the dynamical properties correctly, if the corresponding events are triggered only when the relevant energy becomes higher than a certain threshold value.

We have also introduced a simple form of the bias potential, which is a functional of (part of) the original potential function and does not depend on the details of the potential energy landscape. With the bias potential, we applied the PBD approach to three benchmark problems, including an impurity diffusion process in an amorphous material and folding-unfolding transitions of coarse-grained protein models. We found that the present approach correctly estimated the dynamical properties, indicating that PBD works well for a variety of systems from device materials to biomolecules.

Finally, we note that other forms of the biased potential can also be incorporated into the framework of PBD. Some of them are closely related with the well-established sampling methods such as multicanonical algorithm, Tsallis dynamics, and metadynamics [49]. Thus, incorporating PBD into these 
methods is expected to further propel research on the events that are infrequently observed in MD simulations.

\section{ACKNOWLEDGMENTS}

T.M. thanks Michelle J. S. Spencer for careful reading of the manuscript. This work was supported in part by a Grantin-Aid for Scientific Research from JSPS KAKENHI (Grants No. 15H05563, No. 17K05620, and No. 19H01882). The computations were performed on the computational facilities at the Research Institute for Information Technology, Kyushu University, and at the Research Center for Computational Science, NINS, Japan.

\section{APPENDIX A}

We consider the statistical distribution sampled by the trajectories from the $\mathrm{NH}$ dynamics with the time transformation of Eq. (5). The corresponding EOMs are

$$
\begin{gathered}
\frac{d q_{i}}{d \tau}=\sigma \frac{p_{i}}{m_{i}}, \\
\frac{d p_{i}}{d \tau}=\sigma\left[-\frac{\partial \Phi}{\partial q_{i}}-p_{i} \frac{p_{\eta}}{Q}\right], \\
\frac{d \eta}{d \tau}=\sigma \frac{p_{\eta}}{Q}, \\
\frac{d p_{\eta}}{d \tau}=\sigma\left[\sum_{i=1} \frac{p_{i}^{2}}{m_{i}}-g k_{B} T\right] .
\end{gathered}
$$

The Hamiltonian-like quantity for these dynamical equations is

$$
H_{\mathrm{NH}}=\sum_{i=1}^{3 N} \frac{p_{i}^{2}}{2 m_{i}}+\Phi+\frac{p_{\eta}^{2}}{2 Q}+g k_{B} T \eta,
$$

which is a constant of motion,

$$
\begin{aligned}
\frac{d H_{\mathrm{NH}}}{d t}= & \sum_{i} \dot{p}_{i} \frac{p_{i}}{m_{i}}+\sum_{i} \dot{q}_{i}\left(\frac{\partial \Phi}{\partial q_{i}}\right)+\dot{p}_{\eta} \frac{p_{\eta}}{Q}+g k_{B} T \dot{\eta} \\
= & \sum_{i} \sigma\left(-\frac{\partial \Phi}{\partial q_{i}}\right) \frac{p_{i}}{m_{i}}+\sum_{i} \sigma \frac{p_{i}}{m_{i}}\left(\frac{\partial \Phi}{\partial q_{i}}\right) \\
& +\sum_{i} \sigma\left(-p_{i} \frac{p_{\eta}}{Q}\right) \frac{p_{i}}{m_{i}}+\sigma\left(\sum_{i=1} \frac{p_{i}^{2}}{m_{i}}-g k_{B} T\right) \frac{p_{\eta}}{Q} \\
& +g k_{B} T \sigma \frac{p_{\eta}}{Q}=0 .
\end{aligned}
$$

Meanwhile, the probability density $\rho$ is obtained by solving the following generalized Liouville equation [24]:

$$
\begin{aligned}
\frac{d \rho}{d t} & =-\left(\sum_{i} \frac{\partial}{\partial \Gamma_{i}} \dot{\Gamma}_{i}\right) \rho \\
& =-\rho\left[-\sigma g \frac{p_{\eta}}{Q}+\sum_{i}\left(\frac{\partial \sigma}{\partial q_{i}} \frac{p_{i}}{m_{i}}\right)\right]
\end{aligned}
$$

$$
\begin{aligned}
& =-\rho\left(-g \dot{\eta}+\sum_{i} \frac{\partial \ln \sigma}{\partial q_{i}} \dot{q}_{i}\right) \\
& =-\rho \frac{d}{d t}(g \eta-\ln \sigma),
\end{aligned}
$$

where $\Gamma_{i}$ indicates the phase-space variable including the $\mathrm{NH}$ thermostat variables $\left(\eta\right.$ and $\left.p_{\eta}\right)$. Equation (A7) is immediately solved as

$$
\begin{aligned}
\rho & =C \exp (g \eta-\ln \sigma) \\
& =C \sigma^{-1} \exp (g \eta),
\end{aligned}
$$

where $C$ is a constant. Following the prescription by Tuckerman et al. [50], Eqs. (A5) and (A8) yield the partition function $Z_{\mathrm{NH} \sigma}$,

$$
\begin{aligned}
Z_{\mathrm{NH} \sigma} & =\int d \Gamma \rho \delta\left(H_{\mathrm{NH}}-H_{0}\right) \\
& \propto \int d \Gamma \sigma^{-1} \exp (g \eta) \delta\left(H_{\mathrm{NH}}-H_{0}\right) \\
& \propto \int d \Gamma \sigma^{-1} \exp (g \eta) \delta\left(\eta-\eta_{0}\right) \\
& \propto \int d q d p \sigma^{-1} \exp \left[-\beta\left(\sum_{i=1}^{3 N} \frac{p_{i}^{2}}{2 m_{i}}+\Phi\right)\right] \\
& =\int d q d p \exp \left[-\beta\left(\sum_{i=1}^{3 N} \frac{p_{i}^{2}}{2 m_{i}}+\Phi+\Delta \Phi\right)\right],
\end{aligned}
$$

where $\eta_{0}$ is the solution of $H_{\mathrm{NH}}(\eta)=H_{0}$. This clearly shows that the biased sampling can be performed by solving Eqs. (A1)-(A4). Note that the GIK dynamics can be reformulated to a Hamiltonian dynamics [51,52]; thus the Poincaré transformation is available for constructing the GIK-based gPT dynamics.

\section{APPENDIX B}

In implementing PBD, we need to set the details of the functional form of $\Delta \Phi_{\log }$ [Eqs. (18) and (19)] and the relevant parameters before running the biased MD calculation.

$\Phi(x ; q)$ in Eq. (19) should be part of the whole $\Phi$ (or the whole $\Phi$ itself), which largely depends on $\boldsymbol{x}$. As mentioned in the main text, the torsional degrees of freedom are good candidates for $\boldsymbol{x}$ in biomolecule systems [8,37]. If the whole $\Phi$ consists of several types of potential function (such as harmonic bonding potential, torsion potential, and nonbonded long-range potential, etc.), it is rather straightforward to judge which potential functions should be included in $\Phi(\boldsymbol{x} ; q)$. In the case of condensed materials (such as bulk or surfaces), $\Phi$ often comprises two-body interatomic potentials, indicating that construction of $\Delta \Phi_{\log }$ is again straightforward; the dynamics of some impurities therein, for example, can be easily accelerated by employing their interatomic potentials as $\Phi(\boldsymbol{x} ; q)$. This is exactly what we demonstrate in Sec. III A.

In some cases, however, it may not be clear which degrees of freedom should serve as $\boldsymbol{x}$. Similar problems also arise in free-energy calculations for, e.g., complex nanomaterials, 
wherein suitable collective variables are often required to describe their conformational changes along which free-energy profiles are to be reconstructed. While a satisfactory solution to this problem has not yet been found (and has been a matter of debate for long time), one possible way to systematically find $\boldsymbol{x}$ could be developed by invoking a method of machine learning. Although finding a solution to this problem is beyond the present scope, we believe it deserves future work.

As for the parameter $\varepsilon_{0}$, running a short unbiased MD calculation would be a simple and easy way to estimate its value. If the focused energy-driven event is observed once or a few times in the run, the corresponding energy $[\Phi(\boldsymbol{x} ; q)]$ profile should provide a guide for estimating $\varepsilon_{0}$. It is recommended to set $\varepsilon_{0}$ so as to sufficiently sample the range $\Phi(x ; q)>\varepsilon_{0}$ in the biased MD run.

An appropriate way to find suitable $\alpha$ values can be obtained by noticing the close relationship between the present biased potential [Eq. (18)] and that in accelerated MD [8,9]. In the rest of this section, we discuss their relationship and present a guide to roughly estimate a suitable value of $\alpha$.

Hamelberg et al. [8] introduced a bias potential that can be exploited to enhance sampling rare events in accelerated MD (aMD) simulations. The bias potential $\Delta \Phi_{a}$ in the aMD approach is

$$
\Delta \Phi_{a}=\frac{\left[\varepsilon_{0}-\Phi(\boldsymbol{x} ; q)\right]^{2}}{\left[\gamma+\varepsilon_{0}-\Phi(\boldsymbol{x} ; q)\right]}=\frac{\mu^{2}}{\gamma+\mu},
$$

where $\gamma$ is the parameter that controls the degrees of reduction of the energy barriers (with decreasing $\gamma$, sampling in highenergy ranges is enhanced). The total force acting on the particle $i$ is

$$
\begin{aligned}
-\frac{\partial}{\partial q_{i}}\left(\Phi+\Delta \Phi_{a}\right) & =-\left(\frac{\gamma}{\gamma+\mu}\right)^{2}\left(\frac{\partial \Phi}{\partial q_{i}}\right) \\
& \equiv-\left(\frac{1}{\alpha^{\prime} \mu+1}\right)^{2}\left(\frac{\partial \Phi}{\partial q_{i}}\right),
\end{aligned}
$$

where $\alpha^{\prime}=1 / \gamma$ is introduced to explicitly show the link between $\Delta \Phi_{a}$ and $\Delta \Phi_{\log }$ [Eq. (18)] [we assume that $\Phi(x ; q)$ is coincident with the whole $\Phi$ for simplicity]. Since

$$
-\frac{\partial}{\partial q_{i}}\left(\Phi+\Delta \Phi_{\log }\right)=-\left(\frac{1}{\alpha \mu+1}\right)\left(\frac{\partial \Phi}{\partial q_{i}}\right),
$$

we immediately see that the boost coefficient of the force in aMD [Eq. (B2)] is the square of that of the $\Delta \Phi_{\log }$-based force [Eq. (B3)] if we take $\alpha=\alpha^{\prime}$. This indicates that sampling rare events is significantly enhanced in both approaches. Note, however, that the boosting behavior in aMD would be more sensitive to the parameter $\alpha$ ' due to the squared coefficient, which implies that more careful adjustments of $\alpha^{\prime}(\gamma)$ might be necessary in aMD to properly estimate the correct timescale.

The suitable choice for $\alpha \prime$ is discussed in Ref. [8]. One of the plausible choices is $\gamma \simeq \varepsilon_{0}-\Phi_{\min }$, where $\Phi_{\min }$ is the minimum of $\Phi$ or the lowest value of $\Phi$ sampled in an unbiased MD run. This gives

$$
\alpha^{\prime}=\frac{1}{\left(\varepsilon_{0}-\Phi_{\min }\right)} \equiv \frac{1}{\mu_{\min }},
$$

and thus, the boost coefficient is $1 / 4$ at $\Phi=\Phi_{\min }$. Assuming that this choice is also applied to the bias potential of $\Delta \Phi_{\log }$, we find that suitable $\alpha$ satisfies

$$
\alpha \mu_{\min } \sim 3
$$

or

$$
\alpha \sim \frac{3}{\mu_{\min }} .
$$

This is a rough estimate of $\alpha$ but can be used as a guide to guessing its trial value. In fact, the parameter sets employed in our test calculations presented in the main text are consistent with this choice.

\section{APPENDIX C}

We assume that the number of the phase-space points sampled in the range $\Phi>\varepsilon_{0}$ in the biased MD run is $N_{\varepsilon}^{b}$, while that in the range $\Phi \leqslant \varepsilon_{0}$ in the unbiased MD run is $N_{\varepsilon}^{u}$. If the biased MD trajectory well samples the canonical distribution in the range $\Phi>\varepsilon_{0}$, the correct total time the system stays in the range $\Phi>\varepsilon_{0}$ is $T_{2}=N_{\varepsilon}^{b} d \tau$ (note that $d \tau=d t_{c}$ since $\Delta \Phi=0$ ). Similarly, the total time the system takes $\Phi \leqslant \varepsilon_{0}$ in the unbiased MD run should be $T_{1}^{u}=N_{\varepsilon}^{u} d t_{c}$. We then introduce $N_{\varepsilon_{0}}$ as the number of the phase-space points sampled at $\Phi=\varepsilon_{0}$, which takes $N_{\varepsilon_{0}}^{b}$ and $N_{\varepsilon_{0}}^{u}$ in the biased and unbiased MD run, respectively (normally, $N_{\varepsilon_{0}}^{b} \gg N_{\varepsilon_{0}}^{u}$ ). Let us now imagine a very long "postulated" unbiased MD run on the order of milliseconds or longer, wherein $N_{\varepsilon_{0}}$ happens to be equal to $N_{\varepsilon_{0}}^{b}$. If, then, the actual unbiased MD trajectory $\left(N_{\varepsilon_{0}}=N_{\varepsilon_{0}}^{u}\right)$ samples the correct canonical distribution in the range $\Phi \leqslant \varepsilon_{0}$ (except for the normalization factor), it is reasonable to assume that the total time the system stays in the range $\Phi \leqslant \varepsilon_{0}$ in the postulated long unbiased MD run is given as $T_{1}=\left(\frac{N_{\varepsilon_{0}}^{b}}{N_{\varepsilon_{0}}^{u}}\right) T_{1}^{u}$. Thus, the total time the system evolved along the postulated canonical MD trajectory (that frequently samples the events taking place in $\Phi>\varepsilon_{0}$ ) can be estimated as

$$
T_{c}=T_{1}+T_{2}=C_{\text {boost }} T_{1}^{u}+T_{2},
$$

where

$$
C_{\text {boost }}=\frac{N_{\varepsilon_{0}}^{b}}{N_{\varepsilon_{0}}^{u}} .
$$

When the total time the system takes $\Phi>\varepsilon_{0}$ in the actual unbiased MD run, $T_{2}^{u}$, is approximated as

$$
T_{2}^{u} \sim\left(\frac{N_{\varepsilon_{0}}^{u}}{N_{\varepsilon_{0}}^{b}}\right) N_{\varepsilon}^{b} d t_{c}=\frac{T_{2}}{C_{\text {boost }}}
$$

Eq. (C1) can be simplified as $T_{c}=C_{\text {boost }} T_{c}^{u}$, where $T_{c}^{u}=$ $N^{u} d t_{c}$ and $N^{u}$ is the total number of MD steps in the actual unbiased canonical MD run. In the benchmark calculation for the coarse-grained protein in the main text, we have used this simplified relation. 
[1] M. P. Allen and D. J. Tildesley, Computer Simulation of Liquids, 2nd ed. (Oxford University Press, Oxford, UK, 2017).

[2] M. E. Tuckerman, Statistical Mechanics: Theory and Molecular Simulation (Oxford University Press, New York, 2010).

[3] H. Fujisaki, K. Moritsugu, Y. Matsunaga, T. Morishita, and L. Maragliano, Extended phase-space methods for enhanced sampling in molecular simulations: A review, Front. Bioeng. Biotechnol. 3, 125 (2015).

[4] R. C. Bernardi, M. C. R. Melo, and K. Schulten, Enhanced sampling techniques in molecular dynamics simulations of biological systems, Biochim. Biophys. Acta 1850, 872 (2015).

[5] U. H. E. Hansmann, Y. Okamoto, and F. Eisenmenger, Molecular dynamics, Langevin and hybrid Monte Carlo simulations in a multicanonical ensemble, Chem. Phys. Lett. 259, 321 (1996).

[6] N. Nakajima, H. Nakamura, and A. Kidera, Multicanonical ensemble generated by molecular dynamics simulation for enhanced conformational sampling of peptides, J. Phys. Chem. B 101, 817 (1997).

[7] A. Mitsutake, Y. Sugita, and Y. Okamoto, Generalizedensemble algorithms for molecular simulations of biopolymers, Biopolymers (Pept. Sci.) 60, 96 (2001).

[8] D. Hamelberg, J. Mongan, and J. A. McCammon, Accelerated molecular dynamics: A promising and efficient simulation method for biomolecules, J. Chem. Phys. 120, 11919 (2004).

[9] Y. Miao, F. Feixas, C. Eun, and J. A. McCammon, Accelerated molecular dynamics simulations of protein folding, J. Comput. Chem. 36, 1536 (2015).

[10] C. Tsallis, Computational applications of nonextensive statistical mechanics, J. Comput. Appl. Math. 227, 51 (2009), and references therein.

[11] U. H. E. Hansmann, F. Eisenmenger, and Y. Okamoto, Stochastic dynamics simulations in a new generalized ensemble, Chem. Phys. Lett. 297, 374 (1998).

[12] T. Morishita and M. Mikami, Enhanced sampling via strong coupling to a heat bath: Relationship between Tsallis and multicanonical algorithms, J. Chem. Phys. 127, 034104 (2007).

[13] A. F. Voter, A method for accelerating the molecular dynamics simulation of infrequent events, J. Chem. Phys. 106, 4665 (1997).

[14] A. F. Voter, Hyperdynamics: Accelerated Molecular Dynamics of Infrequent Events, Phys. Rev. Lett. 78, 3908 (1997).

[15] P. Hanggi, P. Talkner, and M. Borkovec, Reaction-rate theory: Fifty years after Kramers, Rev. Mod. Phys. 62, 251 (1990).

[16] R. A. Miron and K. A. Fichthorn, Accelerated molecular dynamics with the bond-boost method, J. Chem. Phys. 119, 6210 (2003).

[17] S. Hara and J. Li, Adaptive strain-boost hyperdynamics simulations of stress-driven atomic processes, Phys. Rev. B 82, 184114 (2010).

[18] D. Hamelberg, T. Shen, and J. A. McCammon, Relating kinetic rates and local energetic roughness by accelerated moleculardynamics simulations, J. Chem. Phys. 122, 241103 (2005).

[19] Y. Xin, U. Doshi, and D. Hamelberg, Examining the limits of time reweighting and Kramers' rate theory to obtain correct kinetics from accelerated molecular dynamics, J. Chem. Phys. 132, 224101 (2010).

[20] T. Morishita, S. G. Itoh, H. Okumura, and M. Mikami, Free energy calculation via mean-force dynamics using a logarithmic energy landscape, Phys. Rev. E 85, 066702 (2012).
[21] T. Morishita, Y. Yonezawa, and A. M. Ito, Free energy reconstruction from logarithmic mean-force dynamics using multiple nonequilibrium trajectories, J. Chem. Theory Comput. 13, 3106 (2017).

[22] S. D. Bond, B. J. Leimkuhler, and B. B. Laird, The NoséPoincaré method for constant temperature molecular dynamics, J. Comput. Phys. 151, 114 (1999).

[23] B. Leimkuhler and S. Reich, Simulating Hamiltonian Dynamics (Cambridge University Press, Cambridge, UK, 2004).

[24] S. Nosé, Constant temperature molecular dynamics methods, Prog. Theor. Phys. Suppl. 103, 1 (1991).

[25] This is also called "Sundman transformation;" see also [26].

[26] K. Zare and V. Szebehely, Time transformations for the extended phase space, Celestial Mech. 11, 469 (1975).

[27] It is easily recognized that one of the necessary conditions for implementing the TST-based method (viz., zero bias at any dividing hypersurface) is closely related with the condition of $\sigma=1$.

[28] S. Nosé, An improved symplectic integrator for Nosé-Poincaré thermostat, J. Phys. Soc. Jpn. 70, 75 (2001).

[29] D. J. Evans, W. G. Hoover, B. H. Failor, B. Moran, and A. J. C. Ladd, Nonequilibrium molecular dynamics via Gauss's principle of least constraint, Phys. Rev. A 28, 1016 (1983).

[30] D. J. Evans and B. L. Holian, The Nose-Hoover thermostat, J. Chem. Phys. 83, 4069 (1985).

[31] D. J. Evans and G. P. Morriss, Equilibrium time correlation functions under Gaussian isothermal dynamics, Chem. Phys. 87, 451 (1984).

[32] Note that the gPT dynamics should also be based on the dynamics that reproduces the "correct" dynamics with $\Delta \Phi=0$.

[33] T. Schneider and E. Stoll, Molecular dynamics study of a three-dimensional one-component model for distortive phase transitions, Phys. Rev. B 17, 1302 (1978).

[34] H. J. C. Berendsen, J. P. M. Postma, W. F. van Gunsteren, A. Di Nola, and J. R. Haak, Molecular dynamics with coupling to an external bath, J. Chem. Phys. 81, 3684 (1984).

[35] U. Doshi and D. Hamelberg, Extracting realistic kinetics of rare activated processes from accelerated molecular dynamics using Kramers' theory, J. Chem. Theory Comput. 7, 575 (2011).

[36] T. Morishita, Fluctuation formulas in molecular-dynamics simulations with the weak coupling heat bath, J. Chem. Phys. 113, 2976 (2000).

[37] D. Hamelberg, C. A. F. de Oliveira, and J. A. McCammon, Sampling of slow diffusive conformational transitions with accelerated molecular dynamics, J. Chem. Phys. 127, 155102 (2007).

[38] Dynamical properties that are evaluated via ensemble averaging are free from this condition; however, $\varepsilon_{0}$ still needs to be set in order to estimate the total time the unbiased system evolved.

[39] F. H. Stillinger and T. A. Weber, Computer simulation of local order in condensed phases of silicon, Phys. Rev. B 31, 5262 (1985).

[40] T. Morishita, Modified velocity scaling scheme for molecular dynamics at constant temperature and/or pressure, Mol. Simul. 29, 63 (2003).

[41] P. H. Hünenberger, Thermostat algorithms for molecular dynamics simulations, Adv. Polym. Sci. 173, 105 (2005).

[42] F. Buda, G. L. Chiarotti, R. Car, and M. Parrinello, Proton Diffusion in Crystalline Silicon, Phys. Rev. Lett. 63, 294 (1989), and references therein. 
[43] M. Isobe, H. Shimizu, and Y. Hiwatari, A multicanonical molecular dynamics study on a simple bead-spring model for protein folding, J. Phys. Soc. Jpn. 70, 1233 (2001).

[44] We chose the following parameter sets that were tuned as the control parameters to exert solvation effects: $\varepsilon=50$ and $e=2.0$ for the first model and $\varepsilon_{c}=50$ and $e_{h b}=3.0$ for the second model, where $\varepsilon_{c}$ is the dielectronic constant and $e_{h b}$ is the parameter (in $\mathrm{kcal} / \mathrm{mol}$ ) that controls the strength of the attractive part in the LJ (hydrophobic) interaction. In addition, an attractive Coulomb interaction with $\varepsilon_{c}=0.0513$ is added to the interaction between the terminal groups in the first model.

[45] B. J. Leimkuhler and C. R. Sweet, A Hamiltonian formulation for recursive multiple thermostats in a common timescale, SIAM J. Appl. Dyn. Syst. 4, 187 (2005).

[46] T. Morishita, From Nosé-Hoover chain to Nosé-Hoover network: Design of non-Hamiltonian equations of motion for molecular-dynamics with multiple thermostats, Mol. Phys. 108, 1337 (2010).

[47] Note that, in general, the massive thermostatting technique does not allow us to evaluate dynamical properties on the "correct" timescale. Here, our purpose is not to correctly predict the timescale of the protein model itself, but rather to see if the PBD approach works for the biomolecule.

[48] Y. Miao, W. Sinko, L. Pierce, D. Bucher, R. C. Walker, and J. A. McCammon, Improved reweighting of accelerated molecular dynamics simulations for free energy calculation, J. Chem. Theory Comput. 10, 2677 (2014).

[49] A. Laio and F. L. Gervasio, Metadynamics: A method to simulate rare events and reconstruct the free energy in biophysics, chemistry and material science, Rep. Prog. Phys. 71, 126601 (2008).

[50] M. E. Tuckerman, Y. Liu, G. Ciccotti, and G. J. Martyna, NonHamiltonian molecular dynamics: Generalizing Hamiltonian phase space principles to non-Hamiltonian systems, J. Chem. Phys. 115, 1678 (2001).

[51] C. P. Dettmann and G. P. Morriss, Hamiltonian formulation of the Gaussian isokinetic thermostat, Phys. Rev. E 54, 2495 (1996).

[52] T. Morishita, Generalized coupling to a heat bath: Extension of the Gaussian isokinetic dynamics and effect of time scaling, J. Chem. Phys. 119, 7075 (2003). 